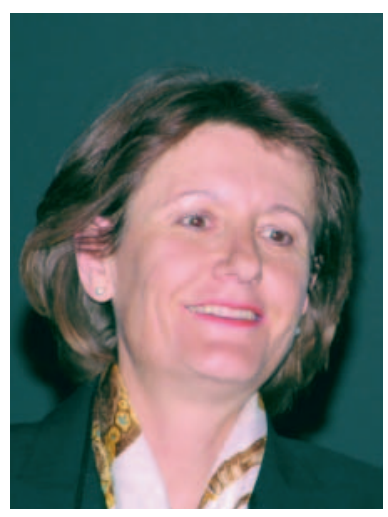

Prof. Dr. pharm. Ursula von Mandach, Zürich

\section{Komplementärmedizin in der Pädiatrie}

Hinter der wachsenden Nachfrage nach komplementärmedizinischer Betreuung mag auch eine gesellschaftspolitische Entwicklung stecken. Es muss jedoch präzisiert werden, dass komplementärmedizinische Ansätze in der medikamentösen Therapie von Kindern ihre Berechtigung haben. In der Pädiatrie eignen sich insbesondere die Phytotherapie und darauf basierende andere komplementärmedizinische Therapien nämlich sehr gut, und zwar aus mehreren rationalen Gründen:

- Sehr viele Phytotherapeutika haben milde Wirkungen oder befinden sich zumindest im mittleren Feld der Skala für die Wirkungsstärke.

- Die milden Wirkungen eignen sich bei vielen Kinderkrankheiten sehr gut als einzige oder additive Behandlung mit geringem Risiko.

- Viele Applikationsformen sind kinderfreundlich und erlangen dadurch bei Kindern und Eltern eine hohe Akzeptanz, wie z.B. Bäder, Sirupe, Umschläge und Tees.

- Phytotherapeutika decken viele Indikationsgebiete in der Pädiatrie ab. So sind sie besonders zur Therapie von Erkältungskrankheiten, Krankheiten des Gastrointestinaltraktes, des psychosomatischen und neurovegetativen Formenkreises sowie verschiedenen Hauterkrankungen indiziert.

- Phytotherapeutika eignen sich auch hervorragend als vorbeugende Massnahme bei Affektionen des Gastrointestinaltraktes und des Immunsystems.

Einige Beispiele von Indikationen sollen in der vorliegenden Ausgabe diskutiert werden. Vielversprechend etwa sind die Ansätze bei der Behandlung von ADHS, nicht zuletzt auch deshalb, weil erkannt wurde, dass das synthetische Methylphenidat $\left(\right.$ Ritalin $\left.^{\circledR}\right)$ unter dem Zwang von Lehrpersonen und Eltern häufig zu rasch und zu wenig selektiv verordnet wird. Dabei wird deutlich, dass komplementärmedizinische Behandlungsmethoden dort einen unschätzbaren Wert haben, wo sie eine Standardtherapie ergänzen oder teilweise erset- zen, also im eigentlichen Sinn komplementär wirken. Bei medikamentösen Therapien erzielen wir dadurch oftmals eine Dosisreduktion und eine Verbesserung der Verträglichkeit sowie letztlich der Compliance. Ob komplementärmedizinische Behandlungsmethoden als Ergänzung oder Alternative gedacht sind, ändert nichts an der Tatsache, dass sie sich erst dann etablieren, wenn die Evidenz ihrer Wirkung erbracht ist. Methoden/Instrumente zu deren Erfassung sind schon lange entwickelt. Wenn wir dabei die Erfahrung ausschliessen, werden wir jedoch bald an unsere Grenzen stossen - an Grenzen, die übrigens auch innerhalb der naturwissenschaftlichen $\mathrm{Me}$ dizin immer wieder erreicht werden. Wie z.B. wollen Sie eine placebokontrollierte Studie in einem Kollektiv von Kranken durchführen, wenn Sie damit einem Teil der Kranken eine effiziente Therapie vorenthalten? Es ist an uns, endlich zu erkennen, dass es wohl sehr ähnliche Werkzeuge gibt, man diese aber nach dem Bedarfsfall variieren kann/muss, um ans Ziel zu gelangen; etwa im Sinne, dass man einen Apfel vorzugsweise mit einem Messer zubereitet als mit einem Löffel, weil es mit dem Messer rascher und sicherer geht, obschon Löffel und Messer beides Essinstrumente sind. Die Erfahrung bleibt ein wichtiges Instrument, das wir auch für die Evidenzerbringung nutzen sollten. Beide, Erfahrung und evidenzbasierte Wirkung, sind deshalb zu kombinieren: Aufgrund der Erfahrung können wir retrospektiv die Anwendung von komplementärmedizinischen Therapien erfassen. Anstatt aus ethischen und anderen Gründen nicht durchführbaren placebokontrollierten Studien können wir prospektive Studien so anlegen, dass sie sich in den Therapieablauf optimal integrieren. Mit solchen Studiendesigns lassen sich Fragen zur Sicherheit (Wirkungen, unerwünschte Arzneimittelwirkungen (UAW), Dosierungen) durchaus beantworten, was ja das Ziel von klinischen, prospektiven Studien ist.

Wir dürfen darauf gespannt sein, inwiefern die von Seiten der Zulassungsbehörden neu geforderten pädiatrischen Kapitel auch bei Nicht-Synthetika ihre Wellen werfen. Die Swissmedic hat in der Verwaltungsverordnung "Anleitung Anforderung an die Arzneimittelinformation von Humanarzneimitteln» und dem dazugehörenden Merkblatt (Erläuterung zur Fachinformation) vom 30.08.2010, insbesondere im Kapitel Dosierungsempfehlungen, obligat Hinweise für die Pädiatrie vorgesehen. Kreativität zur Erstellung der pädiatrischen Prüfpläne wird gefragt sein, um die Empfehlungen auf Evidenz abzustützen. Es ist anzunehmen, dass die Grenzen des Machbaren sichtbar werden - unter Umständen eine Chance, dass die auf den bisherigen naturwissenschaftlichen Werkzeugen abgestützten Therapieverfahren und die komplementärmedizinischen Ansätze dadurch näher zusammenrücken, da sie mit denselben Problemen zu kämpfen haben werden.

Ein frohes, gesundes neues Jahr wünscht Ihnen Prof. Dr. pharm. Ursula von Mandach, Zürich

\section{KARGER}

Fax +497614520714 Information@Karger.de www.karger.com $1015-0684 / 11 / 0231-0005 \$ 38.00 / 0$
Prof. Dr. pharm. Ursula von Mandach, PhD

Perinatale Pharmakologie, Frauenklinik, UniversitätsSpital Zürich

Frauenklinikstrasse 10, 8091 Zürich, Schweiz

Tel. +41 44 255-5136, Fax - 4430

Ursula.vonMandach@usz.ch 